\title{
A Review of Rozeboom's Ideas with an Analysis of Issues in Null Hypothesis Significance Testing
}

\author{
Lexi Brunner
}

Reexamining William Rozeboom's recommendations for the future direction of disciplines such as psychology and philosophy is imminent due to the pressing issues in null hypothesis significance testing (NHST). An overreliance on NHST forms the basis of the replication crisis in psychology. Likewise, the discipline's stringent guidelines on significance levels convey a pressure to publish, which is also significantly contributing to the replication crisis. As researchers' careers are staked on the extent to which they publish, reassessing the fundamental issues with NHST within the context of Rozeboom's ideas is paramount. In this brief review, I focus specifically on Rozeboom's critiques of NHST and on a proposed alternative for evaluating the depth of findings within the framework of theoretical psychology. Thereafter, I compare his foreboding concerns with modern issues facing the validity of psychology and posit Rozeboom prophesized the failings of the discipline years ahead of his time.

Keywords: Theoretical Psychology; NHST; William Rozeboom; P-Hacking; Explanatory Induction 


\section{Overview of Rozeboom's Ideas}

At the invitation of Joseph Royce in 1964, William W. Rozeboom came to the Centre for Advanced Study in Theoretical Psychology at the University of Alberta (Moss \& Kuiken 1998). Born in Iowa, William Rozeboom completed his Ph.D. at the University of Chicago and his postdoctoral fellowship in Minnesota, as well as held teaching positions at both St. Olaf College and Wesleyan University (Aogain 2013). Although he completed his education in the United States, Rozeboom spent ample time in Canada as a professor at the University of Alberta. Through his twenty-nine years at the University of Alberta, Rozeboom bridged the fields of psychometrics, philosophy, and psychology with an impressive 89 publications (Aogain 2013). During the historical time in which Rozeboom was writing, ideas supporting the use of deductive approaches permeated psychology. Specifically, the hypothetico-deductive approach was prominent and endorsed by almost all experimental psychologists in the discipline (Aogain 2013). Hypothetico-deduction is a top-down deductive strategy wherein researchers start with a broad theory and break their theory into specific, falsifiable hypotheses to be tested. Unlike most of Rozeboom's colleagues' captivation with deductive approaches, he instead focused on the philosophy of science and objected to the hypothetico-deductive approach's ignorance of the principal route of routine processing (Aogain 2013). That is, because science progresses cumulatively, theories are already formed via routine collection of data. Consequently, Rozeboom endorsed an explanatory induction (EI) approach to the philosophy of science, instead of a hypothetico-deductive approach. In this paper, I will elucidate Rozeboom's critiques of hypothetico-deductive reasoning with a specific focus on his concerns with the ubiquitous usage of null hypothesis significance tests. Importantly, many of Rozeboom's concerns are still relevant, especially in respect to $p$-hacking and the replication crisis in psychology.

Explanatory Induction

Explanatory induction, as formulated by Rozeboom, is an innate tendency to make interpretations as to why observed data are patterned in certain ways (Aogain 2013). Explanatory induction is conceptualized as an unconscious process that is often overlooked and poorly understood in the philosophy of science. Despite its disregard, Rozeboom contends explanatory induction is potent in the formulation of scientific theories because explanatory induction is naturally innate (Aogain 2013). Accordingly, Rozeboom's conceptualization of scientific inquiry is bottom-up, such that scientists collect data first and then critically reflect on why their theories explain the observed pattern of the data. Hence, in accordance to this inductive approach, it is necessary for theories to be well thought out and analyzed. As explanatory induction occurs naturally in humans, examples of it permeate our day-to-day lives. One such example of EI is inferring a personality trait from an observed behavior. For instance, if on the first day of classes your professor is late, his lecture slides are out of order, and he doesn't keep track of class time, you may infer he is unconscientious. In this way, EI operates unconsciously and automatically to influence one's inferences of behavior.

Notably, Rozeboom extends his argument for EI to the natural sciences. He showcases that it is unfeasible researchers utilized the hypothetico-deductive approach in discovering the basic properties of chemicals' $\mathrm{pH}$ (Aogain 2013). Rather, it is more plausible that chemists were examining chemicals in a lab and uncovered an observable pattern of chemical properties and then conjectured the theory of $\mathrm{pH}$ levels to explain their findings. He extends this logic and applies it to variables which he posits are also inherently conceptual. Variables, he argues, are not created a priori (beforehand), but rather are formulated a posteriori (after the fact) to help explain observable patterns in the world. 


\section{Confirmation Theory}

Rozeboom approaches the confirmation ratio from a Bayesian perspective (Aogain 2013). The confirmation ratio is the degree theory $p$ confirms $q$ proportionate to background knowledge $k$ as a degree of rational probability $(P r)$. Bayesian equations rely on conditional probability wherein one computes the possibility of an event given the probability of something else. These equations are often simplified by the removal of term $k$ (knowledge from relevant sources), however Rozeboom emphatically argues to retain variable $k$ in the Bayesian equation (Aogain 2013). Because he believes knowledge is an inductive accumulation of information, the antecedents $(k)$ are necessarily important in any scientific investigation. Given the importance he places on $k$, theories must be thoroughly thought through before applied to observable patterned data.

Although he is a Bayesian in some regard, he deviates from Bayesians in that he places emphasis on scrupulous consideration of theories rather than examining prior probabilities (base rates), which a Bayesian would likely advocate for instead. In thinking through theories, he suggests examining how conceptual elements are confirmationally related to one another and their implication for study outcomes (Aogain 2013). Hence, the strength of theories rest in those that have attained inductive confirmation, not theories that rely on hypothetico-deductive reasoning. Therefore, Rozeboom does not support the holistic account of hypothetico-deductive reasoning which holds that all components of a theory receive support when any part of them is confirmed. Put in context, this holistic hypothetico-deductive reasoning would suggest for example because one uncovers some empirical support $(p<0.05)$ for the existence of backward conditioning (a component) that this support also holds for the theory of classical conditioning (the whole). Instead, Rozeboom would argue researchers should examine the concepts underlying classical conditioning, with a focus on the subsumed nature of backward conditioning. In doing so, psychologists should scrutinize the pattern of observed data and reconsider the nature of backward conditioning and its relationship to classical conditioning. Therefore, although backward conditioning may receive support in the laboratory, Rozeboom would note this support does not automatically extend to the theory of classical conditioning.

Psychology and Philosophy

Because Rozeboom was also dedicated to the field of philosophy, a brief examination of his ideas about the nature of philosophy and psychology are appropriate. As an avid writer of both disciplines, Rozeboom felt both disciplines could make slight adjustments to unite; he advocated philosophers should spend more time fixating on the method of induction and psychology would benefit from more thorough analysis of concepts (Aogain 2013). As a proponent of EI it is unsurprising Rozeboom suggested these shifts for both disciplines.

Uniquely, Rozeboom proposes psychology faces similar challenges as the natural sciences. Correspondingly, his view is that psychology should be held to the same scientific rigor as the natural sciences. To comply with normal standards of objectivity in science, he endorses the view that psychological research must be based in behavioral data (Aogain 2013). Since his standard for psychology lays in observable behavior, Rozeboom does not differentiate between attributing causes of the observable behavior to mental properties or physical actions. As long as psychology continues to base theories from observable patterns of data, the extent they are confined to physical or mental theories is irrelevant (Aogain 2013). Unlike his lenient position on mentalistic or physical explanations for behavior, Rozeboom takes a stronger stance on attributing behavior to brain mechanisms. He argues implicating the brain in a causal role reduces inquiry of behavior to neurophysiology. Although there is a place for neurophysiology, 
Rozeboom considers it to be irrelevant unless these neuro-descriptions also extend into the domain of psychology (Aogain 2013).

Psychometrics

Rozeboom censures the lack of inductive inference models in psychology by putting blame on psychometricians' focus on formal mathematics (Aogain 2013). He believes mathematical formulas are unwarranted in psychology, because they cannot clarify the nature of theories (Aogain 2013). Moreover, psychologists' desire for quantification is ultimately a result of our need to simplify the complex world. To do so, we create discrete values even though many natural phenomena are continuous. Rozeboom also critiques psychometrics' traditional emphasis on criterion validity over construct validity in the creation of psychometric tests (Aogain 2013). Criterion validity, is the extent a measure is related to an outcome. For instance, one use of criterion validity is in predictive situations such as the extent GRE scores predict performance in graduate school. Alternatively, construct validity is the degree to which a measure actually measures what it purports to measure; for example, an intelligence test measuring one's real level of intelligence. He believes more weight should be placed upon construct validity because it measures what EI deems is important; namely, the extent observable data patterns relate back to theoretical constructs (Aogain 2013). Thus, the observed pattern of intelligence should be highly relatable to the theoretical construct of intelligence. Criterion validity according to Rozeboom should be secondary as results from tests like the GRE are observations that specifically relate to individuals taking the test, rather than to intelligence as a concept.

\section{Common Sense in Psychology}

Common sense and psychology are connected via their reliance on EI (Aogain 2013). Methodologically, Rozeboom bridges the gap between common sense and psychology by positing both share the same mode of explanatory induction, as EI is biologically innate. Explanatory induction's pervasiveness is apparent in common sense via humans' abilities to make theoretical inferences of observable phenomenon. One such example of this is our natural tendency to infer personality traits from others' behaviors. Rozeboom also proposes the natural sciences share a similar inferential structure as both common sense and psychology, thereby further drawing the natural sciences to the human sciences. Thus, common sense, psychology, and the natural sciences all share a common thread of EI, via its innate disposition (Aogain 2013). Accordingly, the distinction between common sense and the sciences is merely a degree of theoretical sophistication. Expanding on this, Rozeboom posits that common sense is extremely useful for the formulation of theories, because even if common sense is incorrect, it leads us to pathways which generate empirically valid theories (Aogain 2013).

Now that Rozeboom's main ideas have been discussed, the remainder of the paper will be dedicated to an in-depth analysis of Rozeboom's ideas around null hypothesis significance testing. Presently, this focus is important as the problems of $p$-hacking and the replicability crisis pose pressing issues for the integrity of psychology.

\section{Null Hypothesis Significance Testing}

Definitions and a Working Example

To simplify statistical jargon, I will describe an example that will continually be referenced to explain the fundamental issues in null hypothesis significance testing. Imagine a researcher decides to investigate whether there is a difference between the conditioning rates of cats and dogs. Before unpacking the results of this hypothetical researcher's study, it is important 
to understand Rozeboom's concerns with null hypothesis significance testing. Rozeboom (1960) vehemently protests scientists' ill-informed reliance on NHST. Recall that null hypothesis significance testing is a form of hypothetico-deductivism as hypotheses are deduced from theory and tested according to pre-identified ranges of acceptance and rejection (Aogain 2013). The test yields a rejection of the null hypothesis when the test statistic falls into the extremes of a distribution; that is, if the value in the distribution appears unlikely due to chance (usually less than a 5\%). If so, the null hypothesis is rejected and the theory it stems from garners more support. The rejection region is predetermined, and accordingly researchers may encounter two types of error when using the null hypothesis significance test (Rozeboom 1960).

Type $I$ error occurs when one rejects the null hypothesis when in fact it is true. For example, the null hypothesis from the working example is that there are no differences in conditioning rates between cats and dogs. If, however the statistical test indicates the null hypothesis is false (there is a difference between conditioning of cats and dogs), when in fact, there is not, the research would suffer from Type I error. Although the data fell in the null hypothesis significance test pre-determined rejection regions, this was in fact a false positive. On the contrary, Type II error occurs when the null hypothesis significance test shows the data are not extreme enough to indicate a rejection of the null hypothesis, when in fact there is a real difference. In terms of the prior example, the case would be that there is a significant difference between the ease of conditioning for cats and dogs, however the significance test failed to pick up on this difference.

Despite the widespread popularity of null hypothesis significance tests of Rozeboom's time (and the present day), this method of statistical analysis as a premise for theoretical support is ultimately flawed. Instead of this binary approach, Rozeboom supported the scrutiny of theoretical components. To illustrate why null hypothesis significance testing is ultimately destructive, Rozeboom (1960) presents four arguments for the reconsideration of these tests' utility.

The Four Issues in Null Hypothesis Significance Testing In The Fallacy of the Null Hypothesis Significance Test, Rozeboom (1960) identifies four shortcomings of the traditional null hypothesis significance test procedure:

(1) Coming back to the earlier example about the differences in conditioning between cats and dogs, suppose the calculated $t$-value from this experiment is 3.20. Yet, imagine that to conclude cats and dogs condition differently (reject the null hypothesis), the $t$-value must be greater than 3.30. Mathematically speaking, the researcher would be beckoned to accept the null hypothesis as their test statistic was not extreme enough to consider rejecting the null hypothesis. Yet, a $t$-value of 3.20 is still quite a large value for this experiment and a value this divergent may be erroneously contained within the acceptance region.

(2) In the traditional null hypothesis significance test, the null hypothesis is set to 0 (customary notation is $\mathrm{H}_{0}=0$ ). In opposition to this, the alternative hypothesis is predetermined to not be $0\left(\mathrm{H}_{\mathrm{A}}<0, \mathrm{H}_{\mathrm{A}}>0\right.$, or $\left.\mathrm{H}_{\mathrm{A}} \uparrow 0\right)$. Rozeboom (1960) questions the assumption as to why the null hypothesis must always be set to 0 . He contends the actual value of $\mathrm{H}_{0}$ can be computed, and in doing so, the acceptable rejection regions would also shift. In other words, by setting an arbitrary number as the null hypothesis, researchers may in turn be doing themselves a disservice as the aforementioned $t$-value of 3.20 could be rejected by a mere change in the predetermined value of the null hypothesis. 
(3) Another principal concern Rozeboom (1960) addresses concerning null hypothesis significance tests lie in their binary outcome; either the null hypothesis is rejected or accepted. Imagine the psychologist conducts another study on the differences in conditioning rates between cats and dogs. He uncovers the null hypothesis is accepted because the test statistic is 2.09 and for the null hypothesis to be rejected, this value must be more extreme than 2.09. Hence, the acceptance region for this study falls between 2.09 and 2.09. This means that any $t$-values between -2.09 and 2.09 are treated equally; they result in confirmation of the null hypothesis. Null hypothesis significance tests treat a diverse range of values with the same outcome, in this case, as the acceptance of the null hypothesis. In other words, there is a drastic difference between obtaining a value of -2.09 and 2.09; in fact, these values are opposite to one another on the number line. By treating all values under the acceptance region the same - accepting the null hypothesis Rozeboom (1960) points out that a value of 2.09 would surely be more similar to 2.10 (in the rejection region) than a value of -2.09 in the acceptance region. However, null hypothesis significance testing places a strict boundary as to what constitutes an acceptance of the null hypothesis and what values are extreme enough to reject, thereby removing any nuances of the observed data.

(4) The confidence level to which we set the standards for acceptance/rejection is also somewhat arbitrary (Rozeboom 1960). If one changes their confidence level to be more at risk for Type I error, their test statistic may accordingly shift from the null hypothesis acceptance towards its rejection. Thus, by merely choosing a riskier confidence level, researchers may find their results change from acceptance of the null (not usually publishable in an academic journal) to rejection of the null hypothesis (publishable). Clearly, Rozeboom (1960) was critiquing the fact that methodological choices in a single experiment can dictate the outcome of not only one's hypothesis, but also the theory for which it stands. Likewise, acceptance or rejection of a proposition should not be definitive, instead it must be interpreted in a continuous way (Rozeboom 1960). Consequently, this all-or-nothing mentality, may erroneously lead researchers to make behavioral adjustments in accordance with their acceptance or rejection of a proposition. For instance, if the working example's null hypothesis is accepted, then in accordance to the hypothetico-deductive approach there is no cause for future investigation. Accordingly, the researcher may no longer investigate conditioning rates of cats and dogs even though classical conditioning is surely more complex than the outcome of one experiment. Despite the researcher not finding significant results, Rozeboom (1960) would argue he should refine his theory with this new information rather than discarding it entirely.

\section{A Proposed Alternative}

The task of the scientist is to infer the extent observed data confer the belief. Rather than the dichotomous decision of rejecting or accepting the null hypothesis, Rozeboom (1960) argues scientists' must more holistically consider whether the observed patterns of data logically support the theory. In other words, he is essentially advocating for the use of an EI approach. In so doing, we should relinquish our usage of null hypothesis significance tests for Bayesian probabilities (Rozeboom 1960). Bayesian probabilities confer an advantage over the standard approach because they allow for a more continuous examination of the data rather than mere acceptance or rejection. Consequently, by eradicating the prominence of null hypothesis significance testing procedures, researchers may create situations where all hypotheses are treated equally (Rozeboom 1960). Put differently, the null hypothesis significance procedure 
necessarily fosters a system whereby the null hypothesis is unfairly advantaged over any alternative hypotheses. Instead of being confined to this type of procedure, a Bayesian approach weights probabilities differently thereby putting all probabilities on par with one another.

Using Bayesian probabilities instead of the standard null hypothesis significance tests was only one of Rozeboom's many suggestions (Rozeboom 1960). Other recommendations include putting more resources into uncovering the differences between a priori and a posteriori probability functions, refining Bayesian theory and/or other suitable approaches for these analyses, reporting confidence intervals, and reducing the grip null hypothesis significance testing has in publication decisions (Rozeboom 1960).

The Issues of $P$-Hacking and the Replication Crisis

As null hypothesis significance testing is still the predominant standard in psychology, so too are the problems that Rozeboom (1960) identified with it. In addition to Rozeboom's critiques about null hypothesis significance testing, $p$-hacking and the replication crisis are also emerging concerns. In response to increasing pressure for publications, researchers have devised clever ways to get their data to reach significance levels. $P$-hacking is one such way; the researcher manipulates his data to reach significance levels without prior hypothesizing an underlying causality. One common approach in $p$-hacking is to run more participants than originally stated as a means of getting the null hypothesis inside the rejection region. Similarly, researchers may also do the opposite to be in the rejection region; they will stop running participants if they reach their desired $t$-statistic earlier than originally conceived.

In a similar vein, not only does $p$-hacking pose a problem for the integrity of psychology, it is also contributing to the replication crisis. In short, the replication crisis is the inability for researchers to replicate prominent findings. Obviously, this also reduces the veracity of the field, as there is uncertainty as to which findings are "real" and which findings surface through experimental sleight-of-hand. In part, this crisis is fueled by the file-drawer problem - the difficulty in publishing null findings. This presents a bias into psychology because only significant findings are disseminated and it also creates an incentive to $p$-hack as often scholars' careers and reputations are staked on their number of publications. Consequently, other researchers are implicated by naively investigating null findings that have already been "filed" away as unsubstantial. Importantly, these unaware researchers not only waste time and resources into such ineffectual investigations, but also their expertise is unwittingly wasted on pointless endeavors in lieu of researching substantial phenomena.

\section{Conclusion}

Although scientists may present their research in a hypothetico-deductive way, Rozeboom advances the idea that EI is ultimately the operator. This bottom-up, data-driven approach he argues for permeates all scientific endeavors. The downfall of psychology may occur in part because of researchers' failure to acknowledge the significance of EI and their erroneous emphatic stance with deduction (Aogain 2013). A consequence of conducting research in a hypothetico-deductive way is the unquestioned, systematic usage of the null hypothesis significance test. Due to its many downfalls as a statistical approach, Rozeboom (1960) contends it is an inappropriate method of inference. Instead, scientific inference should focus on Bayesian probabilities (or something similar) which would ultimately allow for deeper consideration of conceptual ideas. In pursuit of an EI approach, Rozeboom proposes by bringing EI into the spotlight, disciplines such as psychology may be refocused (Aogain 2013). Part of this transition is to recognize the imperative nature of conceptual analysis as the backbone of the discipline. Finally, Rozeboom (1960) briefly mentions journals' stringent guidelines for publishing papers 
to be based on the null hypothesis significance test. Although he doesn't explicitly refer to $p$ hacking and the looming replicability crisis in disciplines like psychology, it is easy to recognize that 50 years later Rozeboom's concerns with null hypothesis significance testing have not shaken the discipline nearly enough.

Acknowledgements: I would like to thank Evan Shillabeer and Dr. Baerdvelt for their comments and advised direction in this review.

Disclosure: There are no potential conflicts of interest.

\section{Appendix}

A Priori: theoretical deduction based on reason rather than empirical observation. A Posteriori: an after-the-fact observation from evidence.

Alternative Hypothesis: the probability that there is a significant difference between the populations.

Bayesian Probability: differential weight of probabilities based on prior probabilities.

Construct Validity: degree to which a measure accurately assesses what it purports to measure.

Criterion Validity: the extent a measure is related to an outcome.

Explanatory Induction: an inductive approach in which data is first collected data and then critically reflected on as to how the theory explains the observed pattern of the data.

File-Drawer Problem: the tendency for positive results to be published and negative results to be filed away.

Hypothetico-Deduction: a deductive approach that breaks a broad theory into specific, falsifiable, and testable hypotheses; Rozeboom criticized this approach.

Null Hypothesis: the probability that there is no significant difference between the populations. $\boldsymbol{P}$-Hacking: the researcher manipulates his data to reach significance levels without prior hypothesizing an underlying causality; a common approach is to stop running participants once the desired significance level is reached.

Replicability Crisis: the inability to replicate prominent findings by subsequent researchers. Type I Error: rejection of the null hypothesis when it is in fact true (false positive).

Type II Error: acceptance of the null hypothesis when it is in fact false (false negative). 


\section{Bibliography}

Aogain, E. M. Scientific Inference: The Myth and the Reality. Dublin: Original Writing Ltd., 2013.

Mos, L., \& Kuiken, D. “Theoretical Psychology at the University of Alberta." History and Philosophy of Psychology Bulletin. 10 (1998): 3-12.

Rozeboom, W. W. "The Fallacy of The Null-Hypothesis Significance Test." Psychological Bulletin. 57 (1960): 416-428. 